\title{
Improving the performance of self-contained cylinder drives with load-holding capabilities
}

\author{
Viktor Donkov $^{1}$, Søren Ketelsen ${ }^{1}$, Torben Andersen ${ }^{1}$, Morten Kjeld Ebbesen ${ }^{2}$, and Lasse Schmidt ${ }^{1}$ \\ ${ }^{1}$ Department of Energy Technology ,Aalborg University, Aalborg, Denmark \\ E-mail: vhd@et.aau.dk \\ ${ }^{2}$ Department of Engineering sciences, University of Agder, Grimstad, Norway
}

\begin{abstract}
A self-contained pump-driven cylinder drive system based on two electric motor and pump (TEMPO) units used for actuation of a two-link, medium-sized knuckle boom crane, is investigated. The TEMPO drive system is equipped with load holding valves and is a closed hydraulic system with a gasless self-pressurizing reservoir. Besides accommodating prescribed safety requirements, the load holding valves allow to reduce energy consumption during standstill. The transition between load holding and motion modes should be unnoticeable for the operator, hence the transition between these modes must be fast, a feature that has not been accomplished in previous attempts. An additional challenge with the system is a constant position error appearing during the locking process. By refining the hydraulic circuit and the control structure, these shortcomings are addressed. A simulation study with a realistic loading cycle is conducted in order to investigate the motion performance of the system. The simulation results demonstrate that the mentioned shortcomings are mitigated, suggesting a potential for future load carrying applications based on the proposed TEMPO drive.
\end{abstract}

Keywords: Compact Drives, Load Holding, Control

\section{Introduction}

Self-contained pump-driven cylinder drive systems offer an alternative to standard hydraulic cylinder systems. Standard hydraulic drives are normally based on a proportional valve which throttles the flow between the pressure source and the hydraulic cylinder. Throttling control has the benefit of being simple and robust, but it reduces the overall efficiency of the system. Pump-driven cylinder drives remove the need for throttling by decentralizing the pressure supply i.e. each cylinder has its own pump which delivers only the flow needed to move the cylinder. Pump-driven cylinder drives have been proposed for knuckle boom cranes in [1]. Furthermore, self-contained pump-driven cylinder drives were proposed in [2]. The system offers energy efficiency improvements over the standard hydraulic system and also offers other benefits such as plug-and-play capabilities i.e. only an electrical connection is needed to the self-contained drive. The two pumps driven by separate electric motors in the TEMPO drive compensate for the different flow requirements due to the cross-section area difference in the differential cylinder. The two inputs also allow for control of cylinder motion and back-pressure separately. Knuckle boom cranes require certain safety features one of which is load holding. In a classical system the safety functionality is achieved with Counter-Balance Valves (CBV). If used in pump-driven cylinder drives the CBV can prevent the recovery of energy [3]. A different system for load holding with TEMPO drives was explored in [4]. The feature allows load holding valves to lock the movement of the cylinder in case of a loss in pressure. The authors of [4] focused on the safety of the system and not on making the locking and unlocking process fast. The load holding valves can also be engaged in order to reduce energy losses during standstill, but in order for this to be done it is desired that the locking process happens as fast as possible. If the locking and unlocking happens sufficiently fast, then it can happen automatically without impeding the work flow of the operator which normally drives such a crane. In order to lock the load holding valves, the pressure in one chamber needs to be reduced rapidly which can cause a deviation in the cylinder's position [4]. A method to address this was proposed in paper [5], but it was only tested for open loop control. In this paper the controller methods from [4] and [6] will be combined to facility faster locking and unlocking of the TEMPO drive proposed in [2]. The error during locking is addressed 
before the valves fully lock through manipulation of the references in the control structure. When the load holding valves are opened rapidly vibrations can appear due to the fast change in system parameters. A similar problem was observed in [6] and a control method based on two controllers which operate at the same time was proposed by the authors. This method has been adapted for the problem at hand. The aim of the study is to reduce the 2 s locking and unlocking time reported in [4]. The target for the locking and unlocking process is set as $250 \mathrm{ms,}$ because on average, reaction time takes between 150 and 300 milliseconds for most humans. The model of the hydraulic system will be discussed in Section 2. A linear model and a coupling analysis is presented in Section 3. The control algorithm and the mode switching is discussed in 4. Simulation results are presented in section 5 . These results are discussed and a conclusion is presented in Section 6.

\section{Model}

A knuckle boom crane is chosen for an example application, because this research is a continuation of the work done in [4]. The example crane used in this paper was also used in papers [1], [4] and [7]. An example crane with a similar scale can be seen in Fig. 1. The mechanical model is based on the Euler-Lagrange method [8], but the actual mechanical model is omitted here for brevities sake. The model can be found in [1] or one of the other papers. For convenience the general length and mass of the crane structure are presented in Tab. 1. Paper [9] shows how the inclusion of the electric motors and pumps needed for a compact drive, do not significantly affect the lifting capability of the crane.

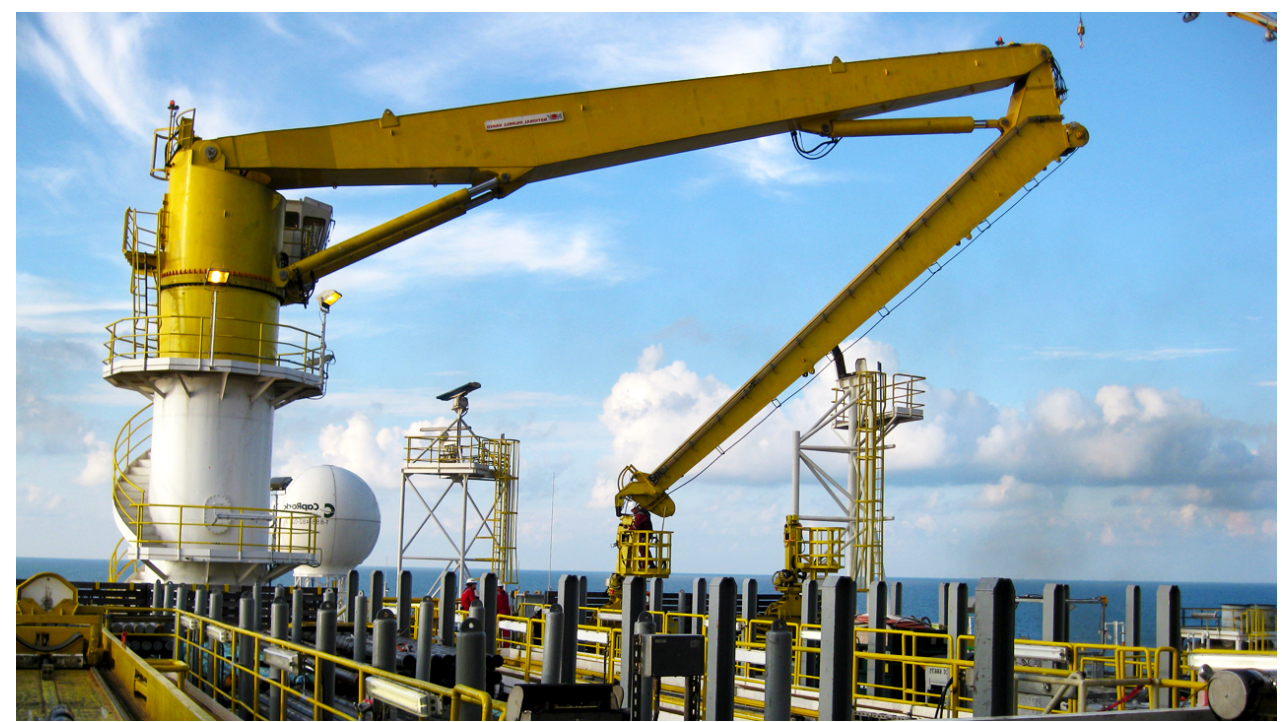

Figure 1: Knuckle Boom Crane example provided by National Oilwell Varco.(C)

Table 1: Sizes for example crane used.

\begin{tabular}{ccc}
\hline Body & Length [m] & Mass [kg] \\
\hline Inner Jib & 13.75 & 6000 \\
Outer Jib & 9.24 & 3300 \\
Cylinder 1 & 2.33 & 1500 \\
Cylinder 2 & 2.84 & 750 \\
\hline
\end{tabular}

The hydraulic circuit which drives the links of the crane can be seen in Fig. 2. One system is needed per link. In this case the focus is on the system driving the main boom of the crane. The TEMPO system is modelled by Eq. (1)-(16). The two valves LHA and LHB are the load holding valves which close if the pressure $P_{\mathrm{C}}$ drops below 10 bar. The inverse shuttle valve IVS always selects the lower of the two pressure $P_{\mathrm{PA}}$ and $P_{\mathrm{PB}}$. The same pressure $P_{\mathrm{C}}$ is also supplied to the charge side of the bootstrap cylinder which provides the pressure on the supply side. Due to the area ratio $A_{\mathrm{C}} / A_{\mathrm{R}}$, a 26 bar pressure on the charge side, results in 2 bar in the reservoir side chamber. Cylinder cross port leakage is assumed negligible for both cylinders, and the following definitions are made $V_{\mathrm{A}}=V_{0 \mathrm{~A}}+A_{\mathrm{A}} X_{\mathrm{P}}, V_{\mathrm{B}}=V_{0 \mathrm{~B}}-A_{\mathrm{B}} X_{\mathrm{P}}, \alpha=A_{\mathrm{B}} / A_{\mathrm{A}}, V_{\mathrm{R}}=V_{0 \mathrm{R}}+A_{\mathrm{R}} Z, V_{\mathrm{C}}=V_{0 \mathrm{C}}-A_{\mathrm{C}} Z, \alpha_{\mathrm{R}}=A_{\mathrm{C}} / A_{\mathrm{R}}$. The volumes $V_{\mathrm{PB}}, V_{\mathrm{PA}}, V_{0 \mathrm{~A}}, V_{0 \mathrm{~B}}, V_{0 \mathrm{R}}$, and $V_{0 \mathrm{C}}$ are constant. 


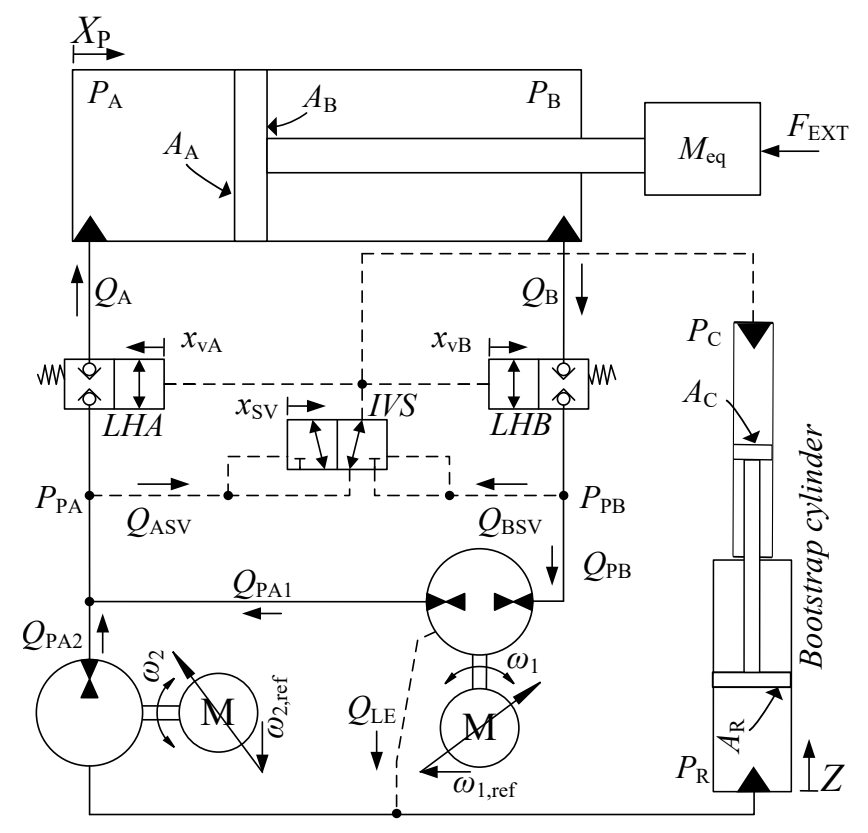

$$
\begin{aligned}
\ddot{X}_{\mathrm{P}} & =\frac{A_{\mathrm{A}}\left(P_{\mathrm{A}}-\alpha P_{\mathrm{B}}\right)-F_{\text {friq }}-F_{\mathrm{EXT}}}{M_{\mathrm{eq}}} \\
\ddot{Z} & =\frac{A_{\mathrm{R}}\left(P_{\mathrm{R}}-\alpha_{\mathrm{R}} P_{\mathrm{C}}\right)-F_{\text {friq, BS }}}{M_{\mathrm{BS}}} \\
\dot{P}_{\mathrm{A}} & =\frac{\beta_{\mathrm{A}}}{V_{\mathrm{A}}}\left(Q_{\mathrm{A}}-\dot{X}_{\mathrm{P}} A_{\mathrm{A}}\right) \\
\dot{P}_{\mathrm{B}} & =\frac{\beta_{\mathrm{B}}}{V_{\mathrm{B}}}\left(\dot{X}_{\mathrm{P}} \alpha A_{\mathrm{A}}-Q_{\mathrm{B}}\right) \\
\dot{P}_{\mathrm{PA}} & =\frac{\beta_{\mathrm{PA}}}{V_{\mathrm{PA}}}\left(Q_{\mathrm{PA} 1}+Q_{\mathrm{PA} 2}-Q_{\mathrm{A}}-Q_{\mathrm{ASV}}\right) \\
\dot{P}_{\mathrm{PB}} & =\frac{\beta_{\mathrm{PB}}}{V_{\mathrm{PB}}}\left(Q_{\mathrm{B}}-Q_{\mathrm{PB}}-Q_{\mathrm{BSV}}\right) \\
\dot{P}_{\mathrm{C}} & =\frac{\beta_{\mathrm{C}}}{V_{\mathrm{C}}}\left(Q_{\mathrm{ASV}}+Q_{\mathrm{BSV}}+\dot{Z} A_{\mathrm{R}} \alpha_{\mathrm{R}}\right) \\
\dot{P}_{\mathrm{R}} & =\frac{\beta_{\mathrm{R}}}{V_{\mathrm{R}}}\left(Q_{\mathrm{LE}}-Q_{\mathrm{PA} 2}-\dot{Z} A_{\mathrm{R}}\right)
\end{aligned}
$$

Figure 2: Asymmetric cylinder controlled by DvSPsystem.

$$
\begin{aligned}
& Q_{\mathrm{PA} 1}=\omega_{1} D_{1}-K_{1}\left(P_{\mathrm{PA}}-P_{\mathrm{PB}}\right)-K_{1}\left(P_{\mathrm{PA}}-P_{\mathrm{R}}\right) \\
& Q_{\mathrm{PB}}=\omega_{1} D_{1}-K_{1}\left(P_{\mathrm{PA}}-P_{\mathrm{PB}}\right)+K_{1}\left(P_{\mathrm{PB}}-P_{\mathrm{R}}\right) \\
& Q_{\mathrm{PA} 2}=\omega_{2} D_{2}-K_{2}\left(P_{\mathrm{PA}}-P_{\mathrm{R}}\right) \\
& Q_{\mathrm{LE}}=K_{1}\left(P_{\mathrm{PA}}-P_{\mathrm{R}}\right)+K_{1}\left(P_{\mathrm{PB}}-P_{\mathrm{R}}\right) \\
& Q_{\mathrm{A}}=x_{\mathrm{vA}} K_{\mathrm{Qv}} \sqrt{\left|P_{\mathrm{PA}}-P_{\mathrm{A}}\right|} \operatorname{sign}\left(P_{\mathrm{PA}}-P_{\mathrm{A}}\right) \\
& Q_{\mathrm{B}}=x_{\mathrm{vB}} K_{\mathrm{Qv}} \sqrt{\left|P_{\mathrm{B}}-P_{\mathrm{PB}}\right|} \operatorname{sign}\left(P_{\mathrm{B}}-P_{\mathrm{PB}}\right) \\
& Q_{\mathrm{ASV}}=\left(1-x_{\mathrm{sv}}\right) K_{\mathrm{Qsv}} \sqrt{\left|P_{\mathrm{PA}}-P_{\mathrm{C}}\right|} \operatorname{sign}\left(P_{\mathrm{PA}}-P_{\mathrm{C}}\right) \\
& Q_{\mathrm{BSV}}=x_{\mathrm{sv}} K_{\mathrm{Qsv}} \sqrt{\left|P_{\mathrm{PB}}-P_{\mathrm{C}}\right|} \operatorname{sign}\left(P_{\mathrm{PB}}-P_{\mathrm{C}}\right) \\
& x_{\mathrm{vA}}=x_{\mathrm{vB}}=\left\{\begin{array}{cc}
0 & \text { for } P_{\mathrm{C}} \leq P_{\mathrm{CR}} \\
\frac{P_{\mathrm{C}}-P_{\mathrm{CR}}}{P_{\mathrm{OP}}-P_{\mathrm{CR}}} & \text { for } P_{\mathrm{CR}}<P_{\mathrm{C}}<P_{\mathrm{OP}} \\
1 & \text { for } P_{\mathrm{C}} \geq P_{\mathrm{OP}}
\end{array}\right. \\
& x_{\mathrm{sv}}=\left\{\begin{array}{cc}
0 & \text { for } P_{\mathrm{PA}}<P_{\mathrm{PB}} \\
0.5 & \text { for } P_{\mathrm{PA}}=P_{\mathrm{PB}} \\
1 & \text { for } P_{\mathrm{PA}}>P_{\mathrm{PB}}
\end{array}\right.
\end{aligned}
$$

$X_{\mathrm{P}}$ is the cylinder piston position, $F_{\text {friq }}$ is the force of friction, $Z$ is the position of the bootstrap cylinder, and $F_{\text {friq,BS }}$ is the friction of the bootstrap cylinder. $P_{\mathrm{A}}, P_{\mathrm{B}}, P_{\mathrm{PA}}, P_{\mathrm{PB}}, P_{\mathrm{C}}, P_{\mathrm{R}}$ are control volume pressures, $Q_{\mathrm{PA} 1}, Q_{\mathrm{PB}}, Q_{\mathrm{PA} 2}$ and $Q_{\mathrm{LE}}$ are pump flows modelled by the Wilson pump model using geometric pump displacements $D_{1}, D_{2}$ and laminar leakage coefficients $K_{1}, K_{2} . Q_{\mathrm{A}}, Q_{\mathrm{B}}, Q_{\mathrm{ASV}}$ and $Q_{\mathrm{BSv}}$ are valve flows modelled by the orifice equation, $x_{\mathrm{vA}}$, $x_{\mathrm{vB}}, x_{\mathrm{sv}}$ are valve poppet positions modelled as quasi static, i.e. no poppet/spool dynamics is included. $P_{\mathrm{CR}}$ and $P_{\mathrm{OP}}$ are valve cracking and full open pressures respectively. The friction model used to determine $F_{\text {friq }}$ can be seen in Eq. (19). The same model with much smaller parameters was used for $F_{\text {friq,Bs. }}$ The friction parameters can be seen in Tab. 2.

$$
F_{\text {friq }}=\left(F_{\mathrm{c}}+\left(F_{\mathrm{s}}-F_{\mathrm{c}}\right) e^{\left(-\operatorname{abs}\left(\dot{X}_{\mathrm{P}}\right) / v_{\mathrm{s}}\right)}\right) \tanh \left(\gamma \dot{X}_{\mathrm{P}}\right)+B_{\mathrm{cyl}} \dot{X}_{\mathrm{P}}
$$

where $F_{\mathrm{c}}$ is the Coulomb friction coefficient, $F_{\mathrm{s}}$ is the static friction coefficient, $v_{\mathrm{s}}$ is the Stribeck velocity, $\gamma$ and the tanh function remove the discontinuity at zero velocity, $B_{\mathrm{cyl}}$ is the viscous friction coefficient. Finally, the effective bulk modulus of the oil air mixture, $\beta_{\mathrm{i}}, i=\{A, B, P A, P B, P I, A C C\}$ is modelled being pressure dependent using Eq. 
(20) according to [10].

$$
\beta_{\mathrm{i}}\left(P_{\mathrm{i}}\right)=\frac{(1-\varepsilon)\left(1+\frac{m\left(P_{\mathrm{i}}-p_{\mathrm{atm}}\right)}{\beta_{\mathrm{F}}}\right)^{-\frac{1}{m}}+\varepsilon\left(\frac{p_{\text {atm }}}{P_{\mathrm{i}}}\right)^{\frac{1}{\kappa}}}{\frac{1-\varepsilon}{\beta_{\mathrm{F}}}\left(1+\frac{m\left(P_{\mathrm{i}}-p_{\mathrm{atm}}\right)}{\beta_{\mathrm{F}}}\right)^{-\frac{m+1}{m}}+\frac{\varepsilon}{\kappa p_{\mathrm{atm}}}\left(\frac{p_{\mathrm{atm}}}{P_{1}}\right)^{\frac{\kappa+1}{\kappa}}}
$$

where $\varepsilon$ is the volumetric air content at atmospheric pressure $\left(p_{\mathrm{atm}}\right), \beta_{\mathrm{F}}$ is the bulk modulus of the pure fluid, $m$ is the pressure dependent bulk modulus gradient of the pure fluid and $\kappa$ is the poly-tropic constant which is set to 1.4, assuming air to behave as an ideal gas and the compression process to be adiabatic. The effective bulk modulus is limited to 7500 bar, to include some mechanical compliance. The permanent magnet synchronous motors are modelled by their current dynamics in the $d q$ rotating reference frame according to [11]:

$$
\begin{aligned}
& \dot{i}_{\mathrm{d}}=\frac{1}{L_{\mathrm{d}}}\left(u_{\mathrm{d}}-R_{\mathrm{s}} i_{\mathrm{d}}+\omega_{\mathrm{re}} L_{\mathrm{q}} i_{\mathrm{q}}\right) \\
& \dot{i}_{\mathrm{q}}=\frac{1}{L_{\mathrm{q}}}\left(u_{\mathrm{q}}-R_{\mathrm{s}} i_{\mathrm{q}}-\omega_{\mathrm{re}} L_{\mathrm{d}} i_{\mathrm{d}}-\omega_{\mathrm{re}} \lambda_{\mathrm{pm}}\right)
\end{aligned}
$$

where $i$ and $u$ are current and voltage respectively, $L$ and $R_{\mathrm{S}}$ are the inductance and the resistance of the stator coils, $\omega_{\mathrm{re}}$ is the electric speed of the rotor shaft, and $\lambda_{\mathrm{pm}}$ is the flux linkage of the permanent magnet in the rotor. The torque produced by these currents are:

$$
T_{\mathrm{e}}=\frac{3}{2} p\left(\lambda_{\mathrm{pm}} i_{\mathrm{q}}+\left(L_{\mathrm{d}}-L_{\mathrm{q}}\right) i_{\mathrm{q}} i_{\mathrm{d}}\right)
$$

where $p$ is the number of pole pairs. This connects the electrical rotor speed $\omega_{\mathrm{re}}$ and the mechanical speed of the motor shaft as $\omega_{\mathrm{re}}=p \omega_{\mathrm{m}}$. This in turn can be modelled by:

$$
\dot{\omega}_{\mathrm{m}}=\frac{T_{\mathrm{e}}-T_{\mathrm{p}}-B_{\mathrm{s}} \omega_{\mathrm{m}}}{J_{\mathrm{s}}}
$$

where $T_{\mathrm{p}}$ applied by the pump, $B_{\mathrm{s}}$ is a friction coefficient, and $J_{\mathrm{s}}$ in the inertia of the rotor, the shaft, and the pump. The sizes of the components can be seen in Tab. 2.

Table 2: Components

\begin{tabular}{cccc}
\hline Component & Size & Component & Size \\
\hline$D 1$ & $26\left[\mathrm{~cm}^{3} / \mathrm{rev}\right]$ & $L_{\mathrm{S}}$ & $1.2[\mathrm{~m}]$ \\
$D 2$ & $28\left[\mathrm{~cm}^{3} / \mathrm{rev}\right]$ & $A_{\mathrm{A}}$ & $0.0616\left[\mathrm{~m}^{2}\right]$ \\
$K_{\mathrm{Qv}}$ & $4.0069 \cdot 10^{-6}\left[\mathrm{~m}^{3} /(\mathrm{sPa})\right]$ & $A_{\mathrm{B}}$ & $0.0302\left[\mathrm{~m}^{2}\right]$ \\
$K_{\mathrm{Qsv}}$ & $1.4086 \cdot 10^{-6}\left[\mathrm{~m}^{3} /(\mathrm{sPa})\right]$ & $A_{\mathrm{C}}$ & $0.0025\left[\mathrm{~m}^{2}\right]$ \\
$P_{\mathrm{CR}}$ & $10[\mathrm{bar}]$ & $A_{\mathrm{R}}$ & $0.0629\left[\mathrm{~m}^{2}\right]$ \\
$P_{\mathrm{OP}}$ & $20[\mathrm{bar}]$ & $B_{\mathrm{S}}$ & $0.01[\mathrm{Nms} / \mathrm{rad}]$ \\
$J_{\mathrm{s}}$ & $0.06\left[\mathrm{~kg} / \mathrm{m}^{2}\right]$ & $L_{\mathrm{q}}, L_{\mathrm{d}}$ & $20.2[\mathrm{mH}]$ \\
$p$ & $3[-]$ & $R$ & $1.30[\Omega]$ \\
$\lambda_{\mathrm{pm}}$ & $0.7857[\mathrm{~Wb}]$ & $B_{\mathrm{cyl}}$ & $30000[\mathrm{Ns} / \mathrm{m}]$ \\
$F_{\mathrm{c}}$ & $1401[\mathrm{~N}]$ & $F_{\mathrm{S}}$ & $409[\mathrm{~N}]$ \\
$v_{\mathrm{s}}$ & $0.0075[\mathrm{~m} / \mathrm{s}]$ & $\gamma$ & $2000[\mathrm{~s} / \mathrm{m}]$ \\
\hline
\end{tabular}

\section{Linear model}

In [2] it was found that the system as presented in the previous section is highly coupled. In order to decouple the motion of the cylinder from the back pressure a number of virtual inputs and outputs need to be designed. The equations for the linear model of system when the load holding valves are open can be defined as Eq. (25) - (29). In these equations the following definitions and assumptions hold $\beta_{0}=\beta_{\mathrm{A}}=\beta_{\mathrm{B}}=\beta_{\mathrm{R}} / \Psi, \rho_{\mathrm{B}}=V_{\mathrm{B}} / V_{\mathrm{A}}$, $\rho_{\mathrm{R}}=V_{\mathrm{R}} / V_{\mathrm{A}}$, and $\rho_{\mathrm{C}}=V_{\mathrm{C}} / V_{\mathrm{A}}$. The assumption of equal bulk modulus in the two control volumes is justified in the fact, that in motion operation mode both chamber pressures are kept at an elevated level (e.g. above 20 bar). $\Psi$ is the ratio between the bulk modulus of the oil in the reservoir side of the bootstrap cylinder and $\beta_{0}$. This parameter is assumed to be known. A study showing how changes in the parameter will affect stability can be seen in [2] and 
a study of how much the parameter actually changes can be seen in [12]. Based on these papers parameter $\Psi$ was calculated at the linearisation point based on (20).

$$
\begin{aligned}
P_{\mathrm{A}} & =\frac{\beta_{0}}{V_{\mathrm{A} 0}}\left(\omega_{1} D_{1}+\omega_{2} D_{2}-\dot{X}_{\mathrm{P}} A_{\mathrm{A}}\right) \\
P_{\mathrm{B}} & =\frac{\beta_{0}}{V_{\mathrm{A} 0}\left(\rho_{\mathrm{B}}+\rho_{\mathrm{C}}\right)}\left(-\omega_{1} D_{1}-\dot{X}_{\mathrm{P}} A_{\mathrm{A}} \alpha+A_{\mathrm{R}} \dot{Z} \alpha_{\mathrm{R}}\right) \\
P_{\mathrm{R}} & =\frac{\beta_{0} \Psi}{V_{\mathrm{A} 0} \rho_{\mathrm{R}}}\left(-\dot{Z} A_{\mathrm{R}}-\omega_{2} D_{2}\right) \\
\ddot{X}_{\mathrm{P}} & =\frac{A_{\mathrm{A}}\left(P_{\mathrm{A}}-\alpha P_{\mathrm{B}}\right)-\dot{X}_{\mathrm{P}} B_{\mathrm{C}}}{M_{\mathrm{eq}}} \\
\ddot{Z} & =\frac{A_{\mathrm{R}}\left(P_{\mathrm{R}}-\alpha_{\mathrm{R}} P_{\mathrm{C}}\right)-\dot{Z} B_{\mathrm{BS}}}{M_{\mathrm{BS}}}
\end{aligned}
$$

\subsection{Input-Output Transformation}

Instead of considering the physical pressure states, [13] found it desirable to formulate an output transformation considering more appropriate pressure states. These virtual pressures were selected to be the virtual load pressure $P_{\mathrm{L}}$, and the level pressure $P_{\mathrm{H}}$ :

$$
P_{\mathrm{L}}=P_{\mathrm{A}}-\alpha P_{\mathrm{B}}, P_{\mathrm{H}}=P_{\mathrm{A}}+H P_{\mathrm{B}}
$$

The level pressure $P_{\mathrm{H}}$ was used to control the pressure in one cylinder chamber to a constant value in [13]. This was done in order to improve the stiffness of the system. In [2] the aim of $P_{\mathrm{B}}$ is twofold. On one hand the chamber pressure is kept constant improving system stiffness, but also the reservoir pressure $P_{\mathrm{R}}$ is controlled in order to prevent cavitation or damage to the pumps' seals. In order to be able to control $P_{\mathrm{R}}$ the following virtual pressures are defined.

$$
P_{\mathrm{L}}=P_{\mathrm{A}}-\alpha P_{\mathrm{B}}, P_{\mathrm{H}}=P_{\mathrm{A}}+H P_{\mathrm{B}}+G P_{\mathrm{R}}
$$

The load pressure is proportional to the piston force and as such closely related to the cylinder motion dynamics. The level pressure will be used to control the pressure in the reservoir side of the bootstrap cylinder. If $H$ and $G$ are defined properly, the virtual level pressure is decoupled from the piston motion/load pressure. This allows the motion of the cylinder and the pressure in the reservoir to be controlled separately. By inserting the non-linear pressure dynamics from Eq. (25), (26) and (27), the dynamics of the virtual outputs become:

$$
\begin{aligned}
\dot{P}_{\mathrm{L}} & =\dot{P}_{\mathrm{A}}-\alpha \dot{P}_{\mathrm{B}} \\
& =\frac{\beta_{0}}{\left(\rho_{\mathrm{B}}+\rho_{\mathrm{C}}\right) V_{\mathrm{A} 0}}\left(-\left(A_{\mathrm{A}} \dot{X}_{\mathrm{P}}-\omega_{1} D_{1}-\omega_{2} D_{2}\right)\left(\rho_{\mathrm{B}}+\rho_{\mathrm{C}}\right)+\left(A_{\mathrm{A}} \dot{X}_{\mathrm{P}} \alpha+A_{\mathrm{R}} \dot{Z} \alpha_{\mathrm{R}}-\omega_{1} D_{1}\right) \alpha\right) \\
\dot{P}_{\mathrm{H}} & =\dot{P}_{\mathrm{A}}+H \dot{P}_{\mathrm{B}}+\dot{H} P_{\mathrm{B}}+G \dot{\mathrm{R}}_{\mathrm{R}}+\dot{G} P_{\mathrm{R}} \\
& =\frac{\beta_{0}}{\left(\rho_{\mathrm{B}}+\rho_{\mathrm{C}}\right) V_{\mathrm{A} 0}} A_{\mathrm{A}}\left(\alpha H-\rho_{\mathrm{B}}-\rho_{\mathrm{C}}\right) \dot{X}_{\mathrm{P}}-\frac{\beta_{0}}{\rho_{\mathrm{R}}\left(\rho_{\mathrm{B}}+\rho_{\mathrm{C}}\right) V_{\mathrm{A} 0}} A_{\mathrm{A}}\left(G\left(\rho_{\mathrm{B}}+\rho_{\mathrm{C}}\right) \Psi-\rho_{\mathrm{R}} \alpha_{\mathrm{R}} H\right) \dot{Z}+ \\
& +f_{1}\left(P_{\mathrm{L}}, P_{\mathrm{H}}, P_{\mathrm{R}}, H, G, \dot{H}, \dot{G}, \omega_{1}, \omega_{2}\right)
\end{aligned}
$$

here $f_{1}\left(P_{\mathrm{L}}, P_{\mathrm{H}}, P_{\mathrm{R}}, H, G, \dot{H}, \dot{G}, \omega_{1}, \omega_{2}\right)$ are the terms not affected by the velocity of the two cylinders i.e. $\dot{X}_{\mathrm{P}}$ and $\dot{Z}$. In order to remove the dependence of $\dot{P}_{\mathrm{H}}$ on $\dot{X}_{\mathrm{P}}$ and $\dot{Z}$, the term $H$ and $G$ are chosen as:

$$
\begin{aligned}
& H=\frac{\rho_{\mathrm{B}}+\rho_{\mathrm{C}}}{\alpha} \\
& G=\frac{\rho_{\mathrm{R}} \alpha_{\mathrm{R}}}{\Psi \alpha}
\end{aligned}
$$

This choice is done according to [2]. That this choice removes the coupling in the system can be seen later in the subsection. With the coupling removed, two virtual inputs are established:

$$
\begin{aligned}
& Q_{\mathrm{L}}=\frac{D_{1}\left(\rho_{\mathrm{B}}+\rho_{\mathrm{C}}+\alpha\right)}{\rho_{\mathrm{B}}+\rho_{\mathrm{C}}} \omega_{1}+D_{2} \omega_{2} \\
& Q_{\mathrm{H}}=\frac{(\alpha-1) D_{1}}{\alpha} \omega_{1}+\frac{\left(\alpha-\alpha_{\mathrm{R}}\right) D_{2}}{\alpha} \omega_{2}
\end{aligned}
$$


Collecting terms in Eq. (32) and (33), and using these definitions of $Q_{\mathrm{L}}$ and $Q_{\mathrm{H}}$ results in the dynamics:

$$
\begin{gathered}
\dot{P}_{\mathrm{L}}=\frac{\beta_{0}}{V_{\mathrm{A} 0}} Q_{\mathrm{L}}+f_{2}\left(P_{\mathrm{L}}, P_{\mathrm{H}}, P_{\mathrm{R}}, \dot{X}_{\mathrm{P}}, \dot{Z}\right) \\
\dot{P}_{\mathrm{H}}=\frac{\beta_{0}}{V_{\mathrm{A} 0}} Q_{\mathrm{H}}+f_{3}\left(P_{\mathrm{L}}, P_{\mathrm{H}}, P_{\mathrm{R}}, \dot{X}_{\mathrm{P}}, \dot{Z}\right)
\end{gathered}
$$

Using the virtual inputs $Q_{\mathrm{L}}$ and $Q_{\mathrm{H}}$ and the virtual outputs $P_{\mathrm{L}}$ and $P_{\mathrm{H}}$ a matrix of transfer functions can be established as:

$$
\left[X_{\mathrm{P}}(s) \dot{X}_{\mathrm{P}}(s) \dot{Z}(s) P_{\mathrm{L}}(s) P_{\mathrm{H}}(s) P_{\mathrm{R}}(s)\right]^{T}=\mathbf{G}_{\mathrm{DC}}\left[\begin{array}{c}
Q_{\mathrm{L}} \\
Q_{\mathrm{H}}
\end{array}\right]
$$

As was mentioned previously the use of the virtual inputs and outputs is warranted by the coupling of the system. In order to check that the new states are decoupled a Relative Gain Array(RGA) analysis is conducted. The RGA number $\left(\mathrm{N}_{\#}\right)$ is defined for diagonal input/output paring in Eq. (41) and off-diagonal pairing in Eq. (42), [14]:

$$
\begin{aligned}
& N_{\# \text { dia }}=\sum_{\mathrm{k}, \mathrm{j}}\left|\mathbf{G} \times\left(\mathbf{G}^{-1}\right)^{T}-\left[\begin{array}{ll}
1 & 0 \\
0 & 1
\end{array}\right]\right| \\
& N_{\# \text { off }}=\sum_{\mathrm{k}, \mathrm{j}}\left|\mathbf{G} \times\left(\mathbf{G}^{-1}\right)^{T}-\left[\begin{array}{ll}
0 & 1 \\
1 & 0
\end{array}\right]\right|
\end{aligned}
$$

where $\mathbf{G}$ is a two by two transfer function, $\times$ is the element-by-element multiplication or Hadamard product. $k, j$ is the number of row and columns in the transfer function matrix. Further details on the RGA and RGA-number can be found in [14] or [15]. If $\mathrm{N}_{\# \text { dia }}$ and $\mathrm{N}_{\# \text { off }}$ attains the values 0 and 4 respectively for all frequencies for a particular input/output pairing, then these can be consider ideally decoupled. In Fig. 3, the coupling analysis for the transfer function Eq. (40) can be seen. The figure shows that the motion of the cylinder can be controlled with the virtual input $Q_{\mathrm{L}}$, while the reservoir pressure can be controlled with the virtual input $Q_{\mathrm{H}}$. No coupling is present.

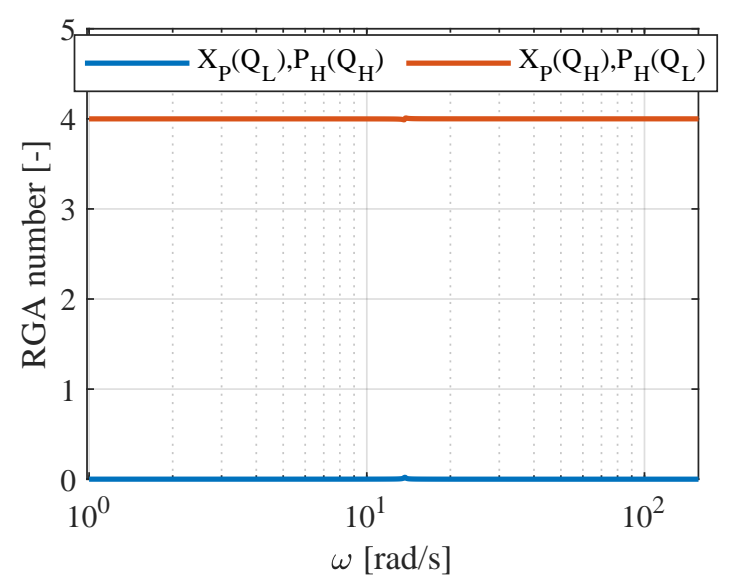

Figure 3: RGA number of motion system

\section{CONTROL}

A block diagram of the control structure can be seen in Fig. 4. The control structure is a cascade controller with a motion controller giving rotational velocity references for the two pumps $\omega_{1, \text { ref }}$ and $\omega_{2 \text {,ref. These references }}$ are then followed by the electric motors which have their own cascade control structure made up from a velocity controller which generates current references $i_{\mathrm{q} 1 \text {,ref }}$ and $i_{\mathrm{q} 2, \text { ref }}$. The $i_{\mathrm{d} 1, \text { ref }}$ and $i_{\mathrm{d} 2 \text {,ref }}$ are selected as zero, because that produces the largest torque. The current references are received by the Field Oriented Controller (FOC) which produces voltage references. Converters are omitted from the model, because of the computational demand which their inclusion produced. A load holding controller controls the pressures $P_{\mathrm{PA}}$ and $P_{\mathrm{PB}}$. The output of the motion controller and the output of the load holding controller are blended together by the mode transition controller. The tuning for all these controllers is shown in the following subsections. 


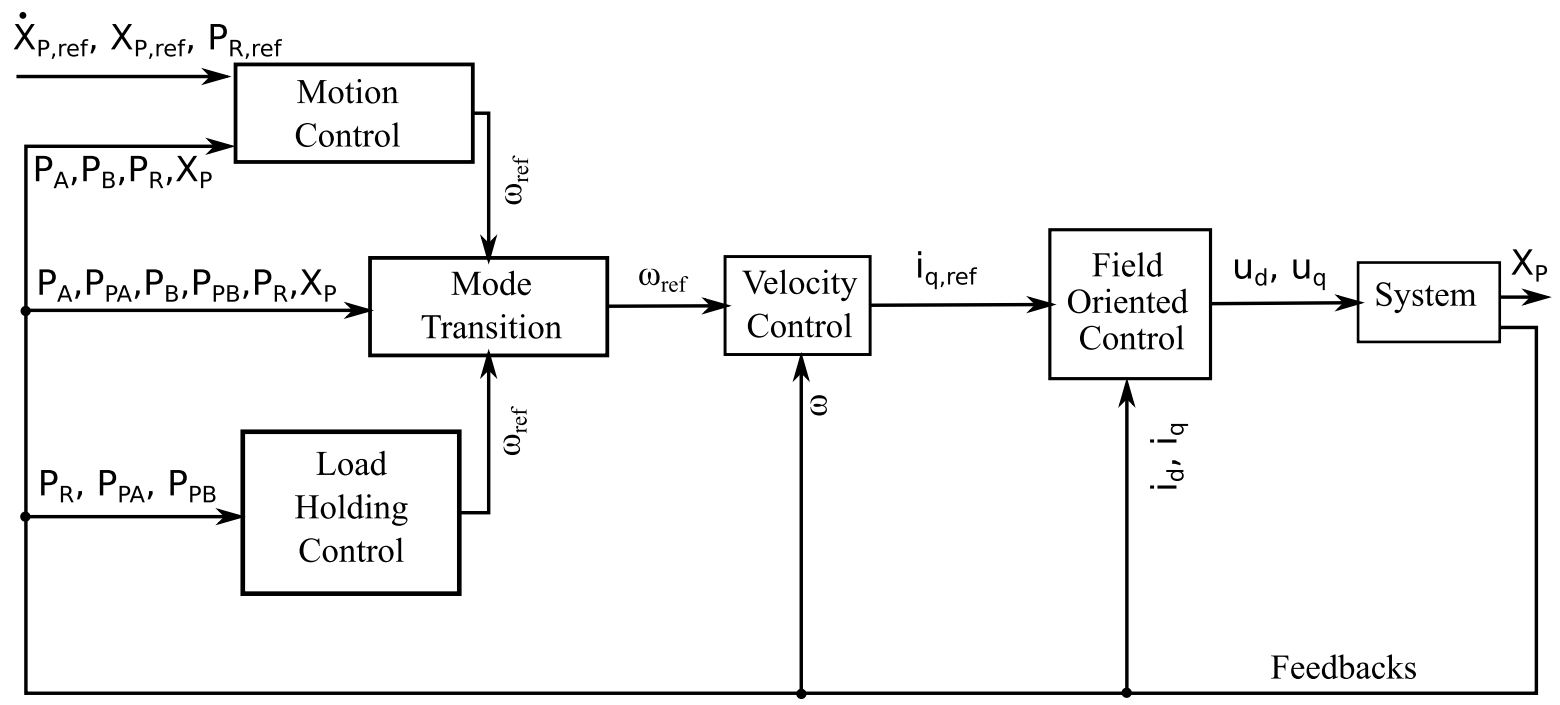

Figure 4: Block diagram of control structure

\subsection{Motion control}

The electric motors are controlled using FOC. A linear model for the current dynamics has been established and a Proportional-Integral (PI) controller is used to obtain a phase margin of $90 \mathrm{deg}$ at $5000 \mathrm{rad} / \mathrm{s}$. This corresponds to a bandwidth of the closed loop of $\approx 800 \mathrm{~Hz}$. This value is chosen to be 10 times slower than the switching frequency of the converter. The bode plot for the open loop of the current dynamics with and without a controller can be seen in Fig. 5. The open loop dynamics of the velocity of the motors can be seen in Fig. 6. A PI controller is used again and the phase margin is selected such that the bandwidth of the motor will be $\approx 80 \mathrm{~Hz}$.

Bode Diagram
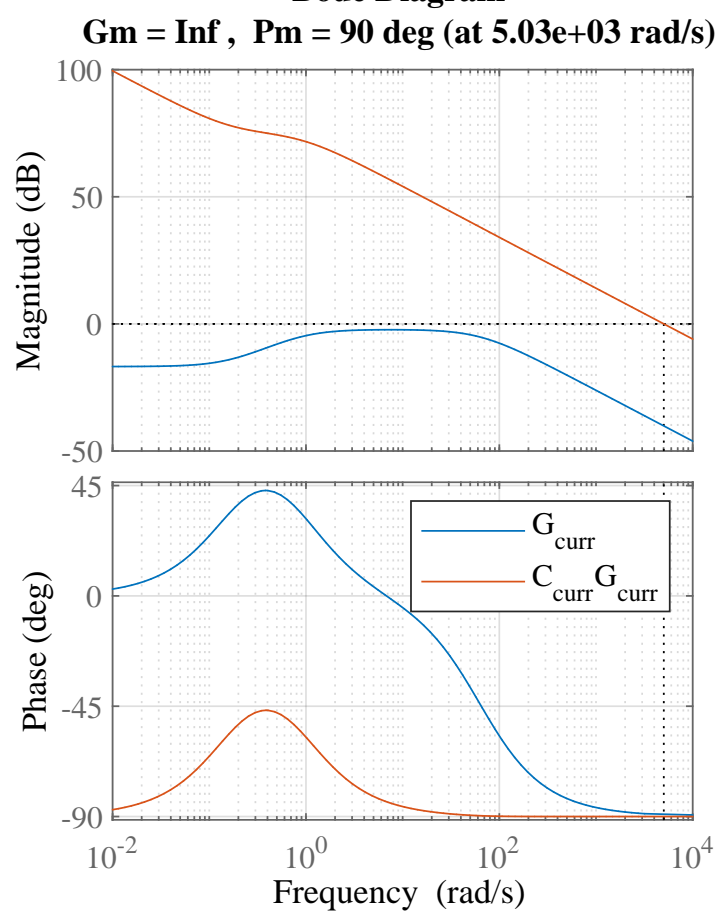

Figure 5: Current controller
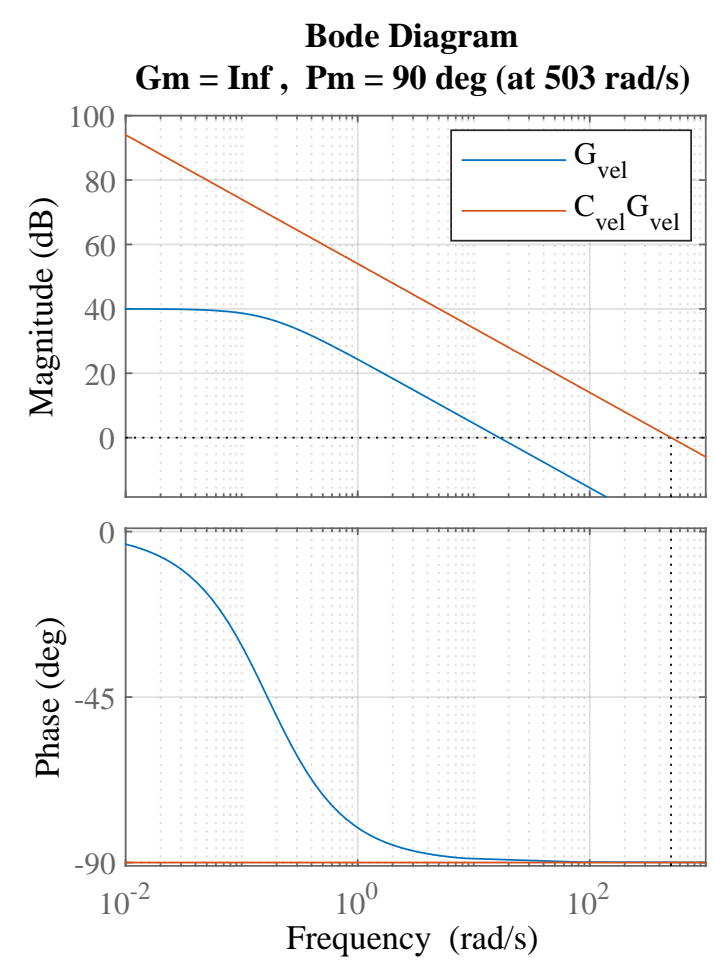

Figure 6: Motor shaft speed controller

A pressure feedback is designed to compensate the position transfer function. This feedback introduces virtual damping into the system and reduces the resonance peak at $13 \mathrm{rad} / \mathrm{s}$. The uncompensated and compensated system can be seen in Fig. 7 


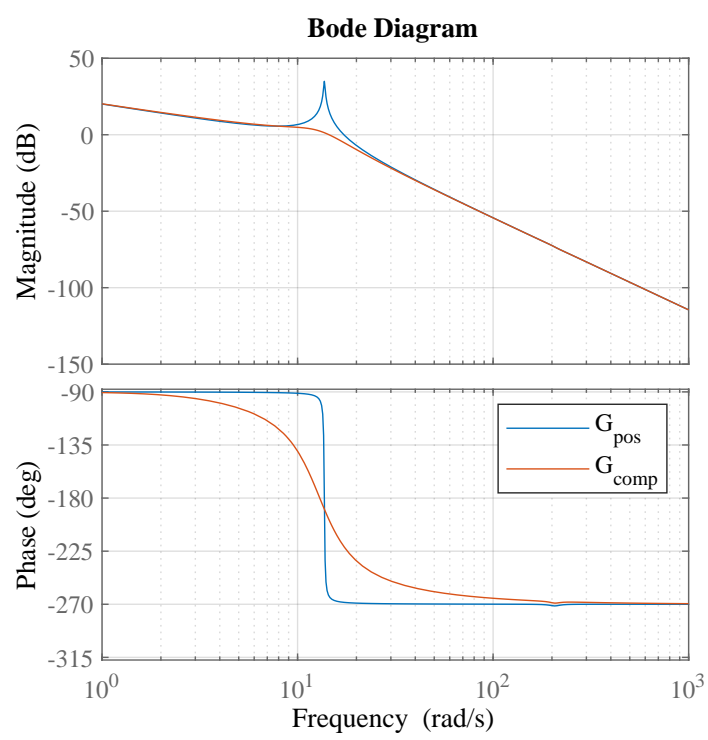

Figure 7: Pressure feedback compensation
Bode Diagram

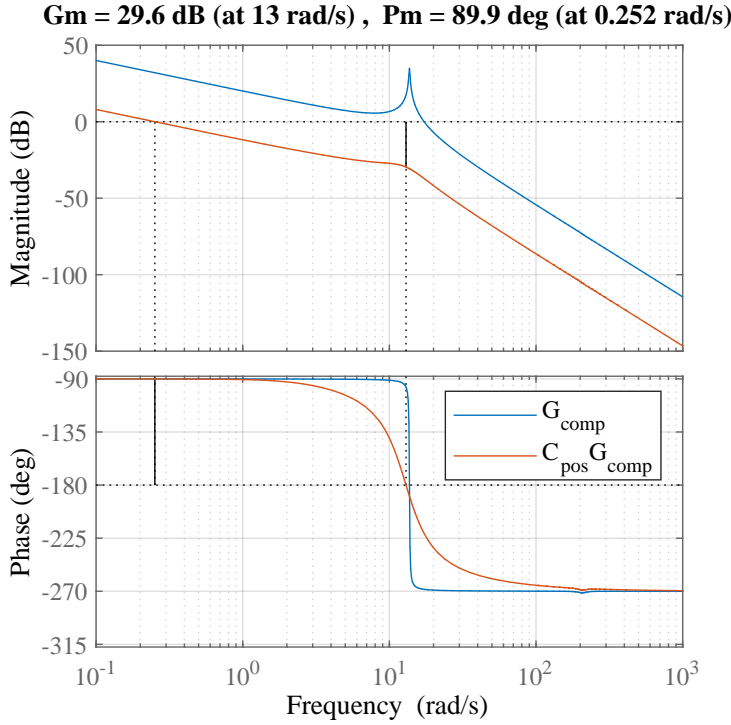

Figure 8: Cylinder position controller

The compensated system is easier to control and a simple proportional controller is used together with a velocity feed-forward. The open loop dynamics can be seen in Fig. 8.

For the $P_{\mathrm{H}}$ controller a proportional controller is used. The open loop dynamics can be seen in Fig. 9.

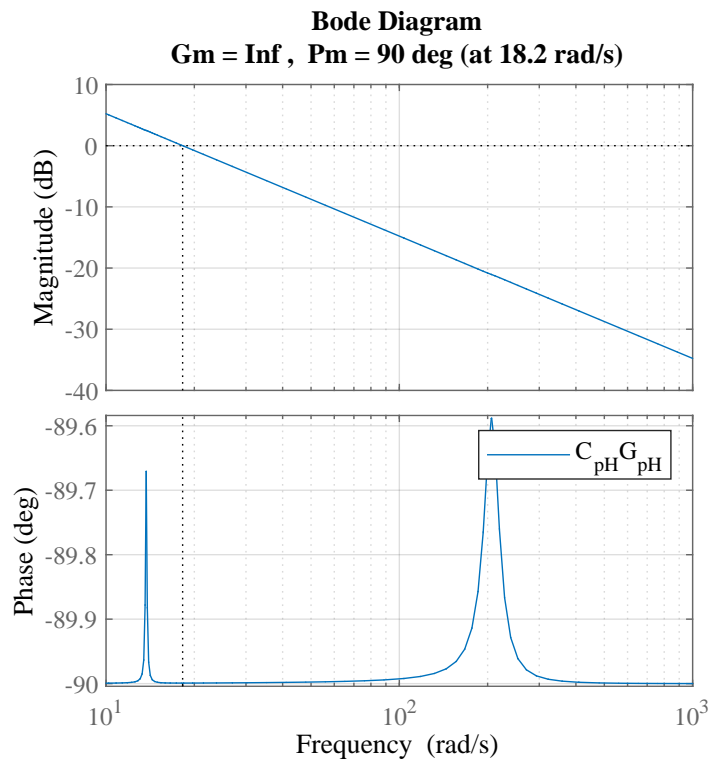

Figure 9: Level pressure controller

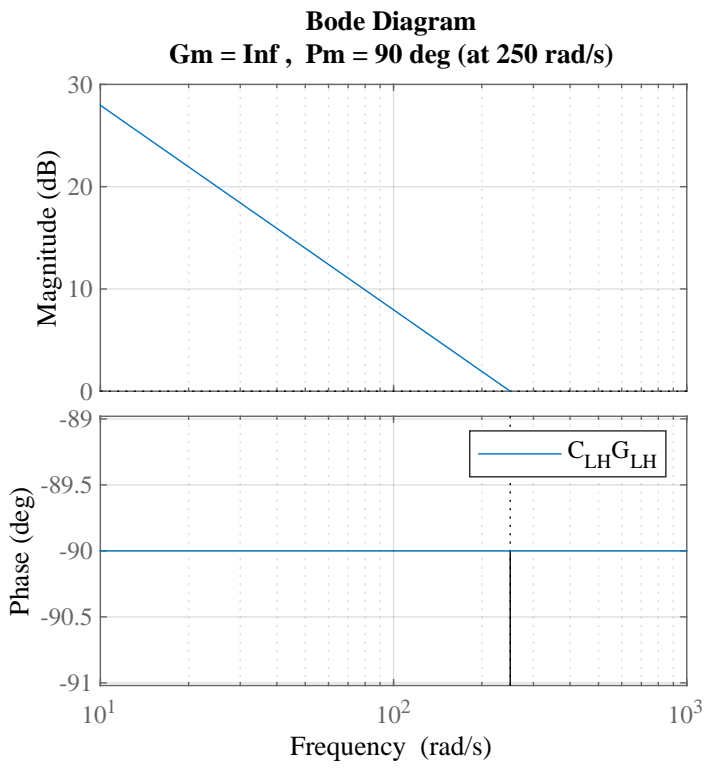

Figure 10: Load holding controller

A proportional controller is also used to control pressure $P_{\mathrm{PA}}$ and $P_{\mathrm{PB}}$ when the load holding valves are engaged. The small volumes $V_{\mathrm{PA}}$ and $V_{\mathrm{PB}}$ result in fast dynamics for the transfer function. Furthermore these controller are tuned much harder, resulting in a higher bandwidth for the closed loop. This is done in order to facilitate the fast locking and unlocking of the load holding valves. The bode plot can be seen in Fig. 10.

\subsection{Transition control}

It was mentioned in the introduction that the aim of this paper is to further the work done in [4]. A smooth transition was prioritised in [4] which resulted in transition times of up to $2 \mathrm{~s}$. Furthermore the piston position was not controlled during the transition period, which resulted in increased position error during the locked period. In 
this paper the transition is achieved through 5 distinct modes. The criteria for switching between modes is shown in Eq. (43).

$$
\text { mode }= \begin{cases}1 & \text { for mode }=0 \& L_{o n}=1 \\ 2 & \text { for mode }=1 \& P_{\mathrm{PB}}<10 \text { bar } \\ 3 & \text { for mode }=2 \& L_{o n}=0 \\ 4 & \text { for } \text { mode }=3 \& \operatorname{abs}\left(P_{\mathrm{A}}-P_{\mathrm{PA}}\right)<l_{\mathrm{T}} \\ 0 & \text { for } \text { mode }=4 \& \operatorname{abs}\left(P_{\mathrm{A}}-P_{\mathrm{PA}}\right)<l_{\mathrm{T}} \& \operatorname{abs}\left(P_{\mathrm{B}}-P_{\mathrm{PB}}\right)<l_{\mathrm{T}} / 100\end{cases}
$$

where $L_{o n}$ is an indicator that load holding should engage and $L_{\mathrm{T}}$ is a threshold value of 1 bar. Mode 0 can be considered normal motion, while mode 2 can be considered locked mode. Modes 1, 3, and 4 are transition modes. In mode 1 the pressure $P_{\mathrm{PB}}$ is reduced until the load holding valves are locked. During mode 3 the pressure $P_{\mathrm{PA}}$ is increased until it matches $P_{\mathrm{PA}}$, so when the load holding valves are opened in mode 4 , the cylinder position does not change. In the current implementation $L_{o n}$ is triggered at certain time stamps and disabled in the same way. In future implementation it can be triggered according to other criteria such as a velocity reference close to zero together with a position error within a preselected limit or an inactivity timer. These could represent that the load has reached its destination. During all modes the transition controller adds the outputs of the motion controller and the load holding controller as seen in Eq. (44). The references sent to these two controllers are changed by the transition controller which facilitates the smooth transition. The two controller which are active at the same time together with filtering of reference pressures removed oscillations during locking and unlocking of a similar system in [6].

$$
u=\omega_{\text {move }}\left(x_{\mathrm{P}}, x_{\text {ref }}, P_{\mathrm{H}}, P_{\text {SetRef }}\right)+\omega_{\text {hold }}\left(P_{\mathrm{PA}}, P_{\mathrm{PAref}}, P_{\mathrm{PB}}, P_{\mathrm{PBref}}\right)
$$

The reference $P_{\mathrm{PB}, \text { ref }}$ changes in the different modes according to:

$$
P_{\mathrm{PB}, \mathrm{ref}}= \begin{cases}P_{\mathrm{B}} & \text { for mode }=0 \\ 1 \text { bar } & \text { for mode }=1 \\ 1 \text { bar } & \text { for mode }=2 \\ 1 \text { bar } & \text { for mode }=3 \\ P_{\mathrm{R}, \mathrm{ref}} / \alpha_{\mathrm{r}} & \text { for mode }=4\end{cases}
$$

During mode $0 P_{\mathrm{PB} \text {,ref }}=P_{\mathrm{B}}$, which means that the load holding controller outputs zero. When load holding is engaged in mode $1, P_{\mathrm{PBref}}$ drops to 1 bar. The controller starts dropping the pressure in the line, which closes $L H B$.

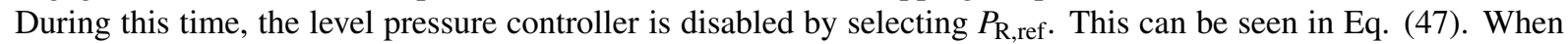
load holding is being disengaged in mode 4 the pressure reference is set to $P_{\mathrm{R}, \text { ref }} / \alpha_{\mathrm{r}}$ so the controller can move $P_{\mathrm{PB}}$ to a value where the level pressure controller can take over again. The reference for $P_{\mathrm{PA} \text {,ref }}$ changes according to:

$$
P_{\mathrm{PA}, \mathrm{ref}}= \begin{cases}1 \text { bar } & \text { for } \text { mode }=2 \\ P_{\mathrm{A}} & \text { else }\end{cases}
$$

In all modes except mode $2 P_{\mathrm{PA}, \text { ref }}$ is equal to the current value of $P_{\mathrm{A}}$. After mode 1 has been engaged and the load holding valves have been closed, maintaining a large pressure in the line serves no purpose. During mode 2 the reference drops to 1 bar, which would reduce the torque on the pumps and motors. Before load holding can be disengaged the pressure in the line has to be raised to the load carrying pressure or the cylinder position will drop as soon as the load holding valves are opened. For this reason $P_{\mathrm{PA}, \text { ref }}$ is set to $P_{\mathrm{A}}$ in mode 3 and mode 4 .

The level pressure controller should produce zero output when load holding is engaged, so $P_{\mathrm{R}, \text { ref }}$ is defined as:

$$
P_{\mathrm{R}, \mathrm{ref}}= \begin{cases}2 b a r & \text { for } \text { mode }=0 \\ P_{\text {set,ref }} & \text { else }\end{cases}
$$

In normal operation the reservoir pressure is controlled to be 2 bar. This corresponds to a $P_{\mathrm{C}}$ of 26 bar. When load holding engages, $P_{\mathrm{R} \text {,ref }}$ is selected as $P_{\text {set,ref }}$, which is calculated according to Eq. (48). This disables the level pressure controller, because it brings the pressure error to zero.

$$
P_{\text {set,ref }}=\frac{P_{\mathrm{H}}-P_{\mathrm{L}}}{\frac{\alpha}{a l p h a_{\mathrm{r}}}+\frac{H}{\text { alpha }}+G}
$$

\section{Results}

The controller described in the previous section was used to follow a $140 \mathrm{~s}$ position trajectory as can be seen in Fig. 11. The figure shows the motion of the cylinder driving the main boom of the crane. Three locking periods are commanded - one from $1 \mathrm{~s}$ to $6 \mathrm{~s}$, a second one from $65.5 \mathrm{~s}$ to $74.5 \mathrm{~s}$, and a third one from 138.5 until the end 
of the simulation. The locking and unlocking cannot be seen clearly in Fig. 11, but can be more easily seen in the error plot seen in Fig. 12 and the plots of the pressures Fig. 13 and Fig. 14. During the second locking period the pressures $P_{\mathrm{A}}$ and $P_{\mathrm{B}}$ oscillate. No flows are coming into or out of the chamber, so these vibrations are caused by the movement of the crane structure. The vibrations can be caused by a modelling error in the friction of the cylinder, which appears to be too small. The problem could also be related to the stiffness of the Matlab solver, but no set of options was found which would provide better results. The first locking process occurs during complete standstill of the cylinder and it can be seen that the pressures do not oscillate. The simulation model should be validated with real hardware, but this was not possible for this study. Laboratory tests are planned in the future. Increasing the gain of the motion controller can be expected to reduce the error over the entire trajectory this resulted in pressure spikes during the unlocking process at $74.5 \mathrm{~s}$.

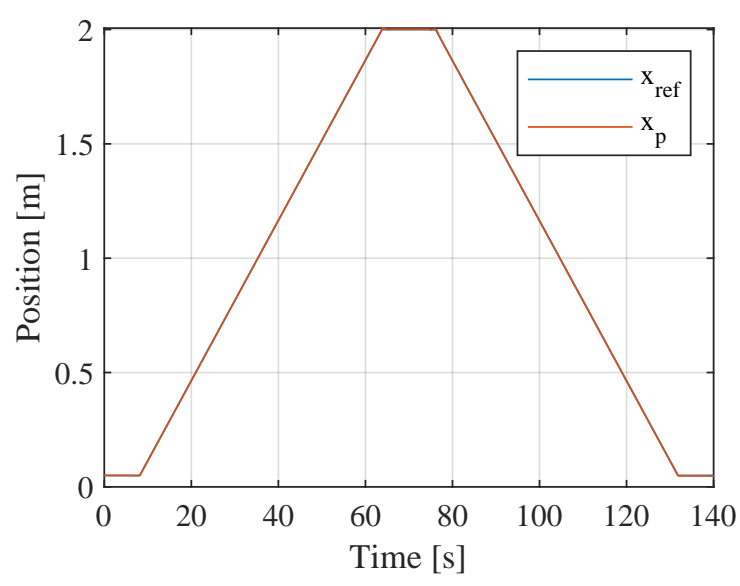

Figure 11: Motion of the cylinder.

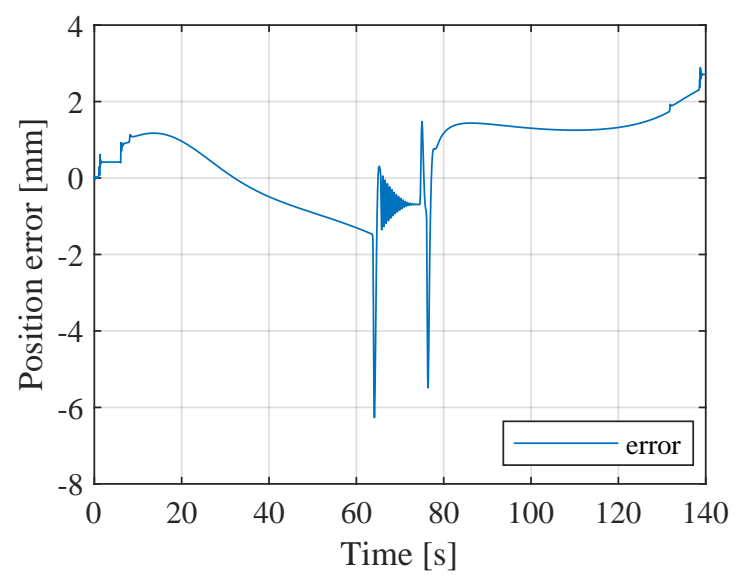

Figure 12: Position error over the trajectory.

During locking the pressure $P_{\mathrm{PB}}$ drops first to close the load holding valves. This can be seen in Fig. 13. As the pressure in chamber B drops the force of the cylinder increases and this would result in cylinder movement. The motion controller tries to counteract this by decreasing the pressure $P_{\mathrm{A}}$. Once the load holding valves are locked $P_{\mathrm{PA}}$ drops as well. A plot showing this can be seen in Fig. 14.

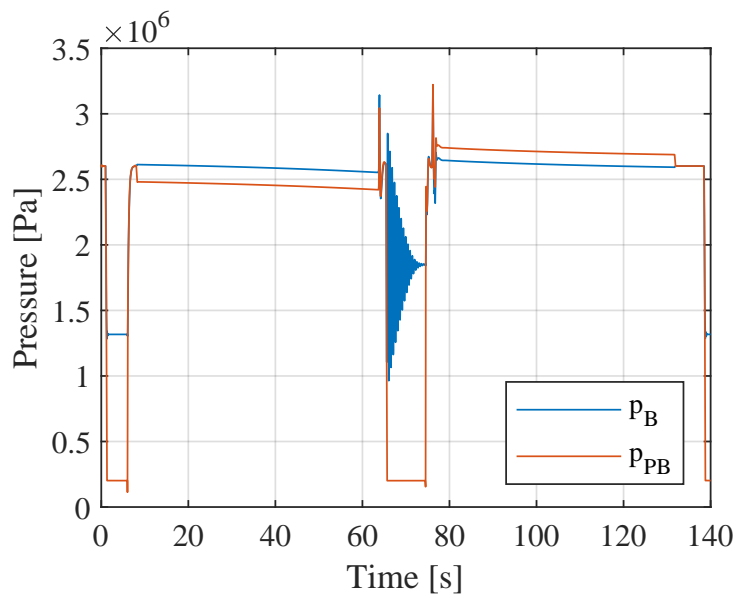

Figure 13: Pressures in line B.

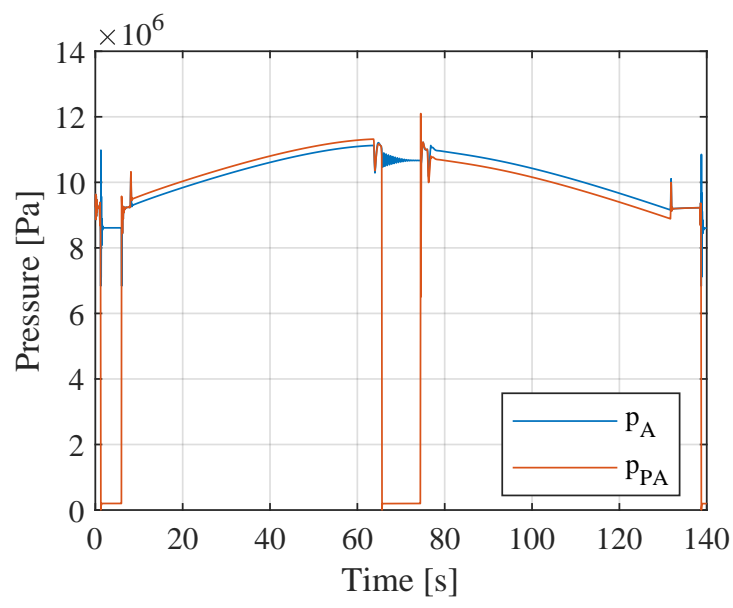

Figure 14: Pressure in line A.

A plot which shows the locking process better can be seen in Fig. 15 and Fig. 16. It takes approximates 250 ms from the locking command until the pressure $P_{\mathrm{PB}}$ drops below 10 bar. The unlocking process happens much slower at approximately $1813 \mathrm{~ms}$. This is due to the condition for switching from mode 4 to mode 0 requiring a very small error between $P_{\mathrm{PB}}$ and $P_{\mathrm{B}}$. Attempting to go into motion mode with a large pressure difference resulted in large outputs from the level pressure controller which the electric motors cannot execute. Reducing the gain of the level pressure controller could reduce this problem, but the large gain is necessary to prevent the level pressure dynamics from becoming unstable at the top of the cylinder. The same instability issue with low level pressure gain was observed in [4] and occurs due to a pole moving into the right half plane. 


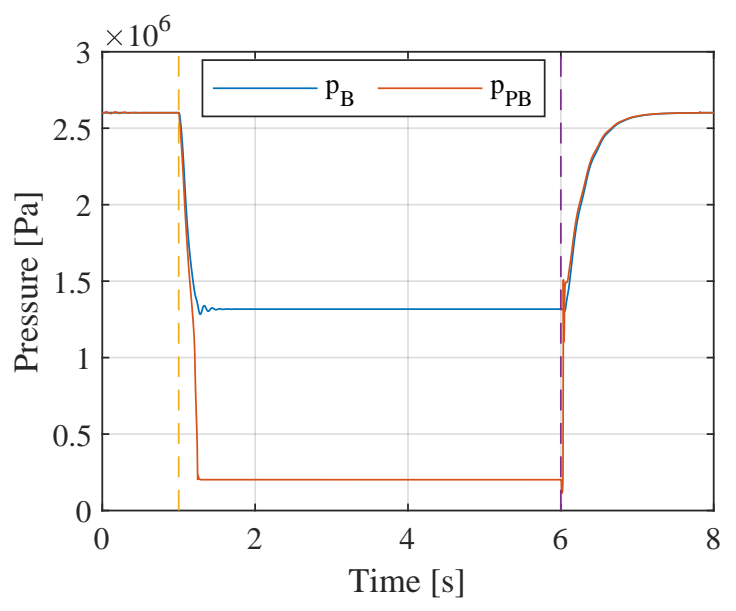

Figure 15: Pressures in line B during load holding.

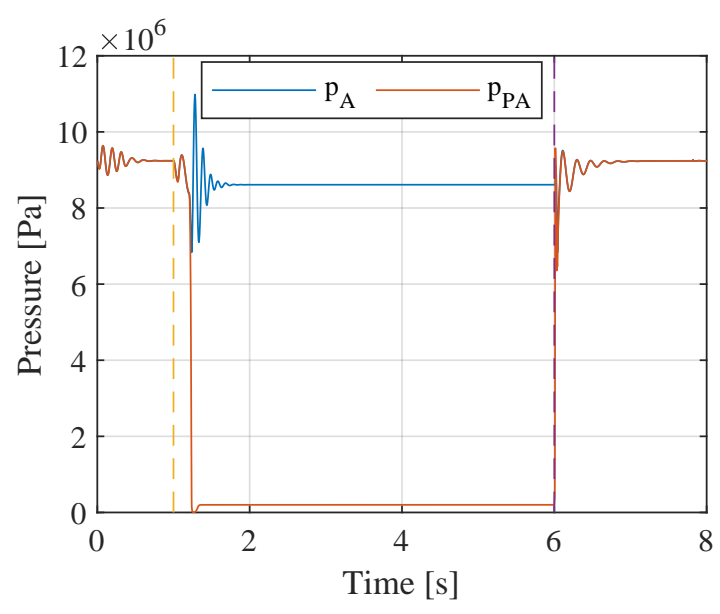

Figure 16: Pressure in line A during load holding.

A surge in the pressure $P_{\mathrm{PB}}$ can be seen at $6.034 \mathrm{~s}$ when the pressure rises above 10 bar which unlocks the load holding valves. The pressure reference $P_{\mathrm{PB} \text {,ref }}$ is filtered with a second order filter according to [6]. Reducing the cut-off frequency of the filter removed this surge but caused other problems and a reduction in locking and unlocking time.

\section{CONCLUSION AND FUTURE WORK}

A simulation study of a knuckle boom crane with self-contained pump-driven cylinder system was conducted. The system has both self-locking capabilities as well as a bootstrap cylinder for a reservoir. The non-linear model is linearised and an input-output transformation is conducted in order to remove the coupling in the system. Controllers for moving, locking and unlocking of the hydraulic cylinder have been developed and explained. The aim of this paper was to reduce the locking and unlocking time from $2 \mathrm{~s}$ reported in [4]. Locking time has been reduced to $250 \mathrm{~ms}$, but unlocking time has only been reduced to $1.8 \mathrm{~s}$. This is due to limitation of the electric motors combined with large variations in system dynamics as the position of the cylinder changes. The unlocking time can be considered unsatisfactory, because it might be too long for the system to be used passively i.e. locking and unlocking every time the velocity reference is zero. For future work the vibrations occurring when the cylinder is locked at its top position need to be investigated. The possibility of choosing different virtual states or using an adaptive controller in order to be able to have a less conservative tuning should also be investigated.

\section{ACKNOWLEDGMENT}

The research in this paper has received funding from The Research Council of Norway, SFI Offshore mechatronics, project number 237896/O30.

\section{References}

[1] Søren Ketelsen, Lasse Schmidt, Viktor Hristov Donkov, and Torben Ole Andersen. Energy saving potential in knuckle boom cranes using a novel pump controlled cylinder drive. 2018.

[2] Søren Ketelsen, Damiano Padovani, Morten Kjeld Ebbesen, Torben Ole Andersen, and Lasse Schmidt. A gasless reservoir solution for electro-hydraulic compact drives with two prime movers. In Fluid Power Systems Technology, volume 83754, page V001T01A038. American Society of Mechanical Engineers, 2020.

[3] Ahmed Imam, Moosa Rafiq, Ehsan Jalayeri, and Nariman Sepehri. Design, implementation and evaluation of a pump-controlled circuit for single rod actuators. In Actuators, volume 6, page 10. Multidisciplinary Digital Publishing Institute, 2017.

[4] Søren Ketelsen, Torben Ole Andersen, Morten Kjeld Ebbesen, and Lasse Schmidt. A self-contained cylinder drive with indirectly controlled hydraulic lock. Modeling, Identification and Control (Online), 2020.

[5] Daniel Hagen and Damiano Padovani. A method for smoothly disengaging the load-holding valves of energyefficient electro-hydraulic systems. 2020. 
[6] Lasse Schmidt, Søren Ketelsen, Damiano Padovani, and Kasper Aa Mortensen. Improving the efficiency and dynamic properties of a flow control unit in a self-locking compact electro-hydraulic cylinder drive. In ASME/BATH 2019 Symposium on Fluid Power and Motion Control. American Society of Mechanical Engineers Digital Collection, 2019.

[7] Viktor Hristov Donkov, Torben Ole Andersen, Henrik Clemmensen Pedersen, and Morten Kjeld Ebbesen. Application of model predictive control in discrete displacement cylinders to drive a knuckle boom crane. In 2018 Global Fluid Power Society PhD Symposium (GFPS), pages 408-413. IEEE, 2018.

[8] Mark W Spong and Mathukumalli Vidyasagar. Robot dynamics and control. John Wiley \& Sons, 2008.

[9] Søren Ketelsen, Torben Ole Andersen, Morten Kjeld Ebbesen, and Lasse Schmidt. Mass estimation of selfcontained linear electro-hydraulic actuators and evaluation of the influence on payload capacity of a knuckle boom crane. In ASME/BATH 2019 Symposium on Fluid Power and Motion Control. American Society of Mechanical Engineers Digital Collection, 2019.

[10] Sunghun Kim and Hubertus Murrenhoff. Measurement of effective bulk modulus for hydraulic oil at low pressure. Journal of Fluids Engineering, 134(2), 2012.

[11] Pragasan Pillay and Ramu Krishnan. Modeling of permanent magnet motor drives. IEEE Transactions on industrial electronics, 35(4):537-541, 1988.

[12] Nikolaj Grønkær, Lasse Nørby Nielsen, Frederik Ødum Nielsen, Søren Ketelsen, and Lasse Schmidt. Multiobjective control of a self-locking compact electro-hydraulic cylinder drive. 2020.

[13] Lasse Schmidt, Morten Groenkjaer, Henrik C Pedersen, and Torben O Andersen. Position control of an over-actuated direct hydraulic cylinder drive. Control Engineering Practice, 64:1-14, 2017.

[14] Sigurd Skogestad and Ian Postlethwaite. Multivariable Feedback Control - Analysis and Design. Wiley, 2. edition, 2005.

[15] Torkel Glad and Lennart Ljung. Control Theory - Multivariable and Nonlinear Methods. Taylor \& Francis, 2000. 